\title{
Frogs and Toads Memory: A Voronoi Twist on the Classic Children's Game
}

\author{
Michael S. Horn and Chia Shen \\ Initiative in Innovative Computing at Harvard University \\ 60 Oxford Street \\ Cambridge, MA 02138 USA \\ Email:mhorn@seas.harvard.edu,chia_shen@harvard.edu
}

\begin{abstract}
In this paper we present a novel twist on the classic children's game, Memory. Here we combine the use of a weighted, centroidal Voronoi diagram and a multitouch tabletop surface to create a board game in which tiles (represented with Voronoi regions) dynamically morph as the game play evolves. This provides a challenge in which players must not only remember the locations of the various tiles, but also track their movements over time.

Ages: 3 - 99

Players: 1 or more
\end{abstract}

Keywords-Voronoi treemaps; application; interaction design; multi-touch tabletop displays

\section{INTRODUCTION}

In the children's game, Memory, a deck of cards is shuffled and arranged face down in a grid. Players take turns flipping two cards at a time. If the flipped cards match (i.e. have identical pictures), the player removes the cards from the table and takes another turn. If the cards don't match, then the player's turn is over, and he or she flips the two cards back over. Game play continues until all of the cards have been matched, and the player with the most cards at the end of the game wins. The basic strategy is for players to try to remember the locations of the cards on the table for future matches-thus the name, Memory. In this paper, we present the design, implementation and preliminary observations of a variation of the Memory game developed using an animated Voronoi tessellation on a multi-touch tabletop.

Multi-touch digital tables, such as Microsoft Surface [4], the DiamondTouch table [3], and the SMART Table [5] are emerging as commercial products available to a broad range of institutions. We are interested in investigating educational and scientific applications of these interactive tables. Such applications might benefit from the friendly, intuitive, and collaborative form factor offered by these multi-touch tables.

\section{Design of AN Animated Memory Game}

We have designed a digitally animated variation of the Memory game on a multi-touch tabletop surface called Frogs $\&$ Toads Memory. In our version of the game, players take turns "flipping" colored tiles on the display by touching them with their fingers (Figure 2). The front faces of the tiles show pictures of frogs and toads from the AmphibiaWeb

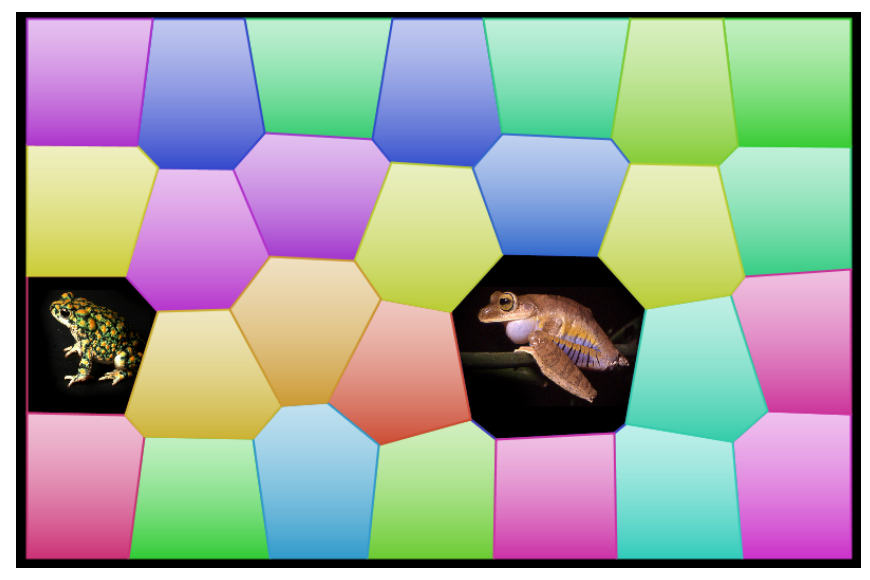

Figure 1. A screen shot from the Frogs \& Toads Memory game. The tiles of the game board are represented with regions from a weighted, centroidal Voronoi diagram. Photo copyrights: 2004 Dr. Axel Kwet (right) and 2001 Adam P. Summers (left).

database [1]. Along with the images, when a card is flipped, we also play audio recordings of calls from the various species.

Instead of presenting players with a rectangular grid of cards (like in the original game), our version uses a tessellation of convex polygons created from a centroidal, additively weighted power Voronoi diagram. Furthermore, the shapes and locations of the tiles slowly shift on the screen as the game is played.

When a player taps one of the tiles on the display, an image is revealed as the corresponding region expands to make room. If the player selects two mismatched tiles, the images are hidden after a short pause, and the tiles return to normal size. If, on the other hand, a player selects two matching tiles, then the images appear to "bubble" up towards the top of the screen and eventually pop. The matching image is then displayed as a thumbnail image along the top of the screen. The remaining tiles on the screen shift and expand to fill the vacant space.

\section{IMPLEMENTATION}

We developed the game using the Java programming language on the Microsoft Surface platform. Microsoft Surface 
is a rear projected tabletop display that uses computer vision to track finger input from multiple simultaneous users.

The game is implemented using a centroidal, additively weighted power Voronoi diagram and the Voronoi treemap algorithm described in [2]. We create $n$ Voronoi regions, one for each game tile, and initialize a set of associated weights $W:=\left\{w_{1}, \ldots, w_{n}\right\}$ with $w_{i}=1$. We also initialize a set of corresponding values $V:=\left\{v_{1}, \ldots, v_{n}\right\}$ with $v_{i}=1$. The Voronoi treemap algorithm iteratively adjusts the weights associated with the Voronoi regions so that the area of each region converges to a value that is approximately equal to $v_{i} A / n$, where $A$ is the total area of the screen.

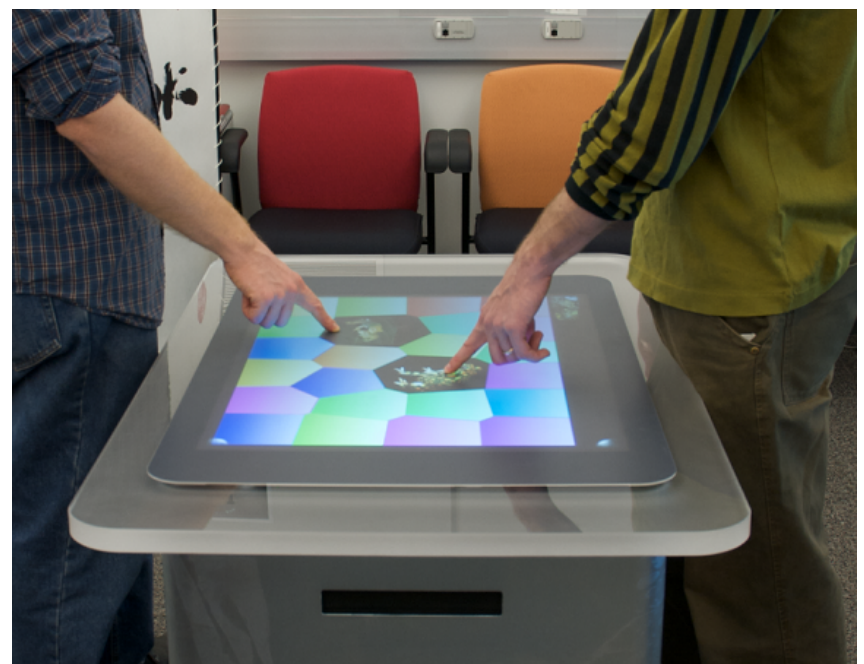

Figure 2. Frogs \& Toads Memory is played on a multi-touch tabletop surface. Players use their fingers to flip game tiles, which are represented with regions of a centroidal, additively weighted power Voronoi diagram.

To animate the tiles, we run an event timer at 30 frames per second. In each frame, we compute a single iteration of the Voronoi treemap algorithm and draw the resulting diagram on the screen. We allow the algorithm to run one step per frame for the duration of the game. Note that the Voronoi tessellation typically stabilizes after one or two seconds.

When a player touches his or her first tile, we increase its corresponding $v_{i}$ value to 2 . The algorithm responds by automatically adjusting the weight of the selected tile so that its area grows to approximately $2 A /(n+1)$. We do the same for the second tile that a player touches. When a tile has been selected, the application draws an image of a frog or toad species inside the Voronoi region (see Figure 1). This image is scaled to fit within the bounding box defined by the Voronoi region.

If the two flipped tiles do not match, the application returns their $v_{i}$ values to 1 after a one second pause. The tiles then return to normal size. If the player successfully matches two tiles, we apply a simulated spring force to their Voronoi generator points to create the illusion that the regions float towards the top of the screen. Ordinarily generator points are moved to the center of mass of their corresponding Voronoi region every iteration; this spring force diverts this "centroidal force" slightly. After a two second pause, the two matching tiles are deleted from the diagram. As the game play continues, the tiles get larger and larger, until only two tiles remain, splitting the screen in half.

\section{OBSERVATIONS}

This game has been shown to many visitors to our lab over the past six months. The subtle movement of the polygons and the unusual, non-rectilinear shapes of the tiles seem to bring forth curiosity and encourage active participation. One unanticipated phenomena is the liveliness and competitiveness that people have shown when playing this digital version. Because our implementation is turnbased, the game rules can be confusing when several people try to touch the table at once. After every successful turn, the regeneration of the Voronoi diagram changes the spatial layout, decreases the number of tiles, and increases this size of the remaining tiles. This, combined with sound effects seems to offer an enhanced psychological reward for the players.

\section{REFERENCES}

[1] AmphibiaWeb: Information on amphibian biology and conservation, 2009. Available from http://amphibiaweb.org. Accessed 27 April 2009.

[2] M. Balzer and O. Deussen. Voronoi treemaps. In INFOVIS '05: Proceedings of the 2005 IEEE Symposium on Information Visualization, pages 49-56, Washington, DC, USA, 2005. IEEE Computer Society.

[3] DiamondTouch, Circle Twelve, Inc., 2009. Available from http://www.circletwelve.com/. Accessed 27 April 2009.

[4] Microsoft Surface, Microsoft Corporation, 2009. Available from http://www.microsoft.com/surface/. Accessed 27 April 2009.

[5] SMART Table, SMART Technologies, 2009. Available from http://www2.smarttech.com/st/enUS/Products/SMART+Table/. Accessed 27 April 2009. 